\title{
Can an observational pain assessment tool improve time to analgesia for cognitively impaired older persons? A cluster randomised controlled trial
}

\author{
Margaret Fry, ${ }^{1,2}$ Lynn Chenoweth, ${ }^{3}$ Glenn Arendts ${ }^{4}$
}

${ }^{1}$ Faculty of Health, University of Technology, Sydney, New South Wales, Australia

${ }^{2}$ Director Research and Practice Development, Northern Sydney Local Health District, Sydney, New South Wales, Australia ${ }^{3}$ Centre for Healthy Brain Ageing, University of New South Wales, Sydney, New South Wales, Australia

${ }^{4}$ School of Medicine, University of Western Australia, Perth, Western Australia, Australia

\section{Correspondence to}

Dr Margaret Fry, Royal North Shore Hospital, Kolling Building, Level 7, Sydney, New South Wales 2065, Australia; margaret.fry@uts.edu.au

Received 19 May 2016 Revised 3 July 2017 Accepted 16 July 2017 Published Online First 5 August 2017
CrossMark

To cite: Fry M, Chenoweth $\mathrm{L}$, Arendts G. Emerg Med J 2018;35:33-38.

\section{ABSTRACT}

Objective The primary objective of the study was to measure the impact of an observational pain assessment dementia tool on time from ED arrival to first dose of analgesic medicine.

Methods A multisite cluster randomised controlled trial was conducted to test the Pain Assessment in Advanced Dementia (PAINAD) tool. Patients aged 65 years or older suspected of a long bone fracture were screened for cognitive impairment using the Six-Item Screening (SIS) tool. Patients scoring 4 or less on SIS (intervention sites) were assessed for pain using PAINAD. Control sites, assessed pain using standard methods. The primary outcome was time to first dose of analgesia and was analysed on an intention-to-treat basis with a sensitivity analysis.

Results We enrolled 602 patients, of which 323 (54\%) were at intervention sites $(n=4)$. The median time to analgesia was $82 \mathrm{~min}$ (IQR 45-151 min). There was no statistically significant difference in median time to analgesia for intervention 83 (IQR 48-158 min) and nonintervention $82 \mathrm{~min}$ (IQR 41-147 min) sites ( $p=0.414)$. After adjusting for age, fracture type, arrival mode and triage category, there remained no significant difference in time to analgesia ( $\mathrm{HR} 0.97,95 \% \mathrm{Cl} 0.80$ to 1.17 , $p=0.74$ ). Of the 602 patients enrolled, 273 actually had cognitive impairment. A sensitivity analysis demonstrated patients at intervention sites received analgesia $13 \mathrm{~min}$ sooner (90 vs $103 \mathrm{~min}, \mathrm{p}=0.91$ ).

Conclusion Use of the PAINAD was not associated with a shorter time to analgesia, although there was a clinically important but non-significant improvement in the cognitively impaired patient group. Further research is needed to address this clinically important and complex issue.

\section{OBJECTIVE}

In line with an ageing population, the number of people presenting to the ED with cognitive impairment is increasing. ${ }^{1}$ Approximately $28 \%$ of ED patients aged 65 years or more have cognitive impairment associated with dementia, delirium and neurological conditions. ${ }^{2}$ Among a number of clinical issues, the ED care of older people with cognitive impairment experiencing pain is suboptimal. ${ }^{3-5}$

We have previously shown that cognitively impaired older people with a long bone fracture wait significantly longer for commencement of analgesia in ED. ${ }^{4}$ ED clinicians, and in particular nursing staff, are the first responders to all patients, and pain assessment is part of this

\section{Key messages}

What is already known on this subject

- People with cognitive impairment wait longer for analgesia compared with other ED groups. Pain assessment tools have been shown to improve time to analgesia in such patients in non-ED settings.

\section{What this study adds}

- In this randomised clustered intervention trial, the use of the Pain Assessment in Advanced Dementia, a pain assessment tool for cognitively impaired adults, did not have a statistically significant impact on time to analgesia for the group as a whole, although a sensitivity analysis of only those with cognitive impairment showed a potentially clinically useful (but non-significant) difference. We suspect that there are additional workload and human factors in the ED that need to be considered in improving the delivery of analgesia to this population.

response. However, ED staff have identified a lack of confidence and familiarity with pain assessment in people with cognitive impairment, especially in those with dementia. ${ }^{6}$ Available guidelines indicate that timely analgesia is important, recognised as a human right and should be provided for those in severe pain within $20 \mathrm{~min}^{7}$ or within a maximum of $60 \mathrm{~min}^{2}$ from time of arrival in ED.

Many researchers ${ }^{8-11}$ advocate for the use of observational pain assessment tools for people with dementia. Observational pain assessment tools, such as the Pain Assessment in Advanced Dementia (PAINAD), can improve pain recognition and intensity measurement by as much as $25 \%-42.5 \% .^{12}$ As a result, groups such as the US Medical Directors Association $^{11} 1314$ and the American Pain Society ${ }^{15}$ advocate for the adoption of PAINAD within clinical practice.

The most common form of ED assessment of pain is patient self-report using a numerical verbal scale. ${ }^{16}$ However, an inability to communicate pain intensity for people with significant cognitive impairment means clinicians rely often on their own subjective assessment, which has been shown to be unreliable. ${ }^{6}{ }^{12}$ To improve pain assessment in these patients, observational pain scales have been developed, where an observer relies on 


\begin{tabular}{|c|c|c|c|c|}
\hline & 0 & 1 & 2 & Score \\
\hline $\begin{array}{l}\text { Breathing } \\
\text { Independent of } \\
\text { vocalisation }\end{array}$ & Normal & $\begin{array}{l}\text { Occasional laboured } \\
\text { breathing. Short period } \\
\text { of hyperventilation }\end{array}$ & $\begin{array}{c}\text { Noisy laboured } \\
\text { breathing. Long period of } \\
\text { hyperventilation. } \\
\text { Cheyne-stokes } \\
\text { respirations }\end{array}$ & \\
\hline $\begin{array}{c}\text { Negative } \\
\text { Vocalisation }\end{array}$ & None & $\begin{array}{l}\text { Occasional moan or } \\
\text { groan. Low level speech } \\
\text { with a negative or } \\
\text { disapproving quality }\end{array}$ & $\begin{array}{l}\text { Repeated troubled calling } \\
\text { out. Loud moaning or } \\
\text { groaning. Crying }\end{array}$ & \\
\hline $\begin{array}{c}\text { Facial } \\
\text { Expression }\end{array}$ & $\begin{array}{l}\text { Smiling, or } \\
\text { inexpressive }\end{array}$ & $\begin{array}{l}\text { Sad. Frightened. } \\
\text { Frown }\end{array}$ & Facial grimacing & \\
\hline $\begin{array}{c}\text { Body } \\
\text { Language }\end{array}$ & Relaxed & $\begin{array}{l}\text { Tense. Distressed } \\
\text { pacing. Fidgeting }\end{array}$ & $\begin{array}{l}\text { Rigid. Fists clenched, } \\
\text { knees pulled up. Pulling } \\
\text { or pushing away. Striking } \\
\text { out }\end{array}$ & \\
\hline Consolability & $\begin{array}{l}\text { No need to } \\
\text { console }\end{array}$ & $\begin{array}{l}\text { Distracted or reassured } \\
\text { by voice or touch }\end{array}$ & $\begin{array}{l}\text { Unable to console, } \\
\text { distract or reassure }\end{array}$ & \\
\hline
\end{tabular}

Figure 1 PAINAD components and scoring system.

psychological and physical pain cues in lieu of patient self-report to rate pain intensity. ${ }^{5}$ This is widely used in the preverbal paediatric ED population. For people with dementia, one such scale is the PAINAD score. ${ }^{17}$ When using PAINAD, an observer assigns a score of $0-2$ across five domains to rate the patient's pain intensity on a scale of $0-10$ (figure 1 ). PAINAD is a reliable indicator of pain intensity ${ }^{12}$ and assesses physiological and psychological pain symptoms providing clinicians with a systematic and structured approach to assessing pain. Psychometric testing of PAINAD demonstrates strong internal consistency $(\mathrm{r}=0.80-0.85)$, internal reliability $(\alpha=0.90)$, concurrent validity $(\mathrm{r}=0.73-.97)$ and inter-rater reliability $(\mathrm{r}=0.75-0.97) .^{13} 18-22$

PAINAD has been validated for use in several clinical settings. ${ }^{13} 23$ However, the scale has never been trialled in the ED setting. The aim of this research was to test whether the use of PAINAD would improve time to analgesia in hospital EDs for older people with cognitive impairment.

\section{DESIGN}

\section{Study design and setting}

A multicentre cluster randomised controlled trial (registration ACTRN 12613000997752) was conducted in eight metropolitan EDs in Sydney, Australia during the period of this study. The study period was March 2013 to June 2015 and during this time period there were $340891 \mathrm{ED}$ presentations.

Approval was obtained from all ethics and governance committees (LNR/12/HAWKE/416 Dec 2012). This study was conducted in accordance with the Declaration of Helsinki. The reporting of this study adheres to the Consort 2010 cluster randomised controlled trial checklist.

\section{Randomisation}

EDs were randomised to receive the intervention (PAINAD) or usual care (figure 2). Australian EDs level 5 (urban district hospitals) and 6 (trauma major referral hospitals) are comparable with respect to attendance patterns, clinical leadership positions, undergraduate/postgraduate training, staff education programs, information systems, resources and staffing. Sydney metropolitan EDs self-selected for study inclusion. The eight EDs were placed into four pairs, pairing the two most closely matching EDs by characteristics such as annual number of presentations and hospital status (trauma major referral hospital or urban district hospital). The lead investigator with an independent witness randomised sites to the intervention or control using a balanced computer coin toss randomisation process.

\section{Study participants}

Male and female patients aged 65 years or more with cognitive impairment and a clinically suspected acute long bone fracture were eligible for enrolment. Suspected long bone fracture was chosen because it is recognised as a uniformly painful condition for which analgesia should be offered in the ED. Patients with suspected long bone fracture, 65 years or more, were triaged as usual. Exclusion criteria: patients were excluded if they met any of the following criteria: a) Australasian Triage Scale category 1 (resuscitation case); b) polytrauma; c) systolic BP $<90 \mathrm{~mm} \mathrm{Hg}$ and d) non-English-speaking patient with no interpreter available. The definition of cognitive impairment used for the study was a Six-Item Screening (SIS) score of 4 or less assessed by the nurse on ED arrival. ${ }^{24}$

Data collection included patient demographics (age, gender); clinical information (time of arrival to the ED, triage code, SIS, doctor seen by time, analgesics prescribed and time, discharge, diagnostic code and disposition). PAINAD scores were collected from intervention sites.

\section{Sample size}

An audit of two participating sites prior to the commencement of the study revealed a mean time to analgesia in patients with dementia with long bone fracture of $185 \mathrm{~min}$ (SD $167 \mathrm{~min})$. The planned sample size of 615 patients (rounded to 300 per group and 75 per site) was based on an intraclass coefficient of 0.05 , $\alpha=0.05 \%$ and $80 \%$ power to detect a mean reduction of $60 \mathrm{~min}$ in the time to analgesia with the intervention. A 60 min cut-off was selected as this is a recommended ED clinical indicator. ${ }^{2}$

\section{Interventions}

Prior to the trial, an education programme was delivered across all sites and included dementia pain signs and symptoms, screening for cognitive impairment using the SIS and study protocol and data collection sheet. ${ }^{17}$ At intervention sites PAINAD education was added. The staff at intervention sites were not blinded to the intervention but were not informed about the primary outcome 


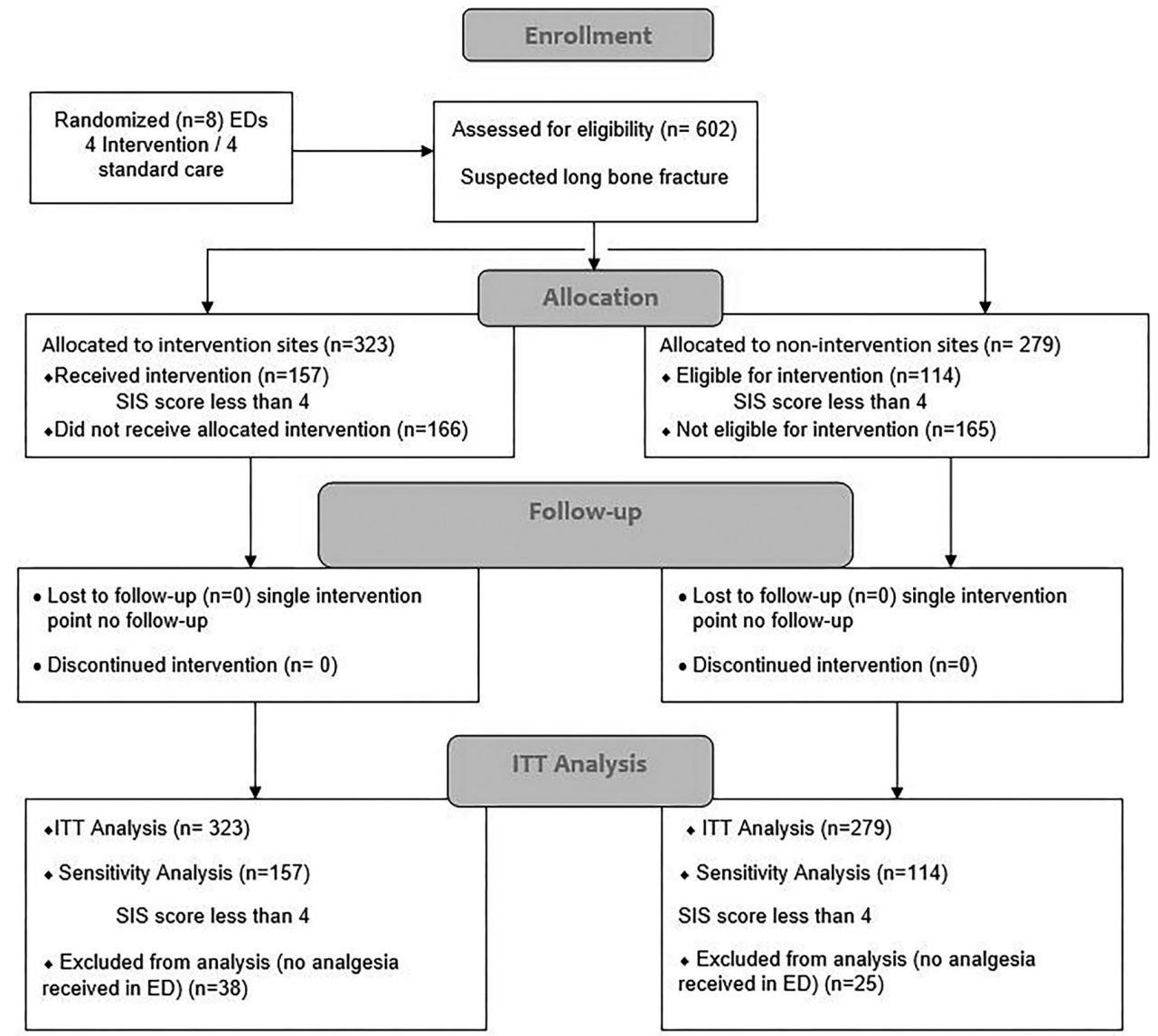

Figure 2 Randomisation and screening process. ITT, intention to treat; SIS, Six-Item Screening tool.

measure of the research in order to minimise the likelihood of bias. To accommodate staff rotations and annual leave, the programme was repeated every 4-6 weeks by either the lead investigator or site champion. Nurse managers and lead clinicians played a vital role in championing the trial across sites and regular in-service meetings were organised to facilitate education and study engagement.

Across all sites, the bedside nurse screened patients for cognitive impairment using the SIS prior to a routine pain assessment. In the non-intervention sites, pain assessment was performed according to usual care. In Australian EDs, a verbal rating scale or a visual analogue scale is the usual pain assessment method for adults. ${ }^{25}$ At intervention sites, a SIS score of 4 or less required the nurse to use the PAINAD to assess pain.

\section{Primary outcome}

Time from ED arrival to first dose of analgesic medicine administered. Analgesia included oral or parenteral medication. Data were collected by manually examining the patient's medical and e-Health records.

\section{Secondary outcomes}

Proportion of patients administered pain medication within $60 \mathrm{~min}$; proportion of patients receiving no analgesia comparing time of arrival with medicine's documentation.

\section{Analysis}

The main analysis conducted was intention-to-treat (basis) with a sensitivity analysis including only those patients in which cognitive impairment was documented and verifiable. Descriptive statistics were used to describe the study population. The
Mann-Whitney $U$ test was used to determine the primary outcome and Pearson's $\mathrm{X}^{2}$ test to determine differences in proportions for secondary outcomes. We then used a Cox regression analysis for time to analgesia and binary logistic regression for the proportion of patients receiving pain medication within $60 \mathrm{~min}$, with both adjusting for confounders of age, fracture type, arrival mode and triage category. Statistical analysis was conducted with IBM SPSS V.21 software.

\section{RESULTS}

\section{Characteristics of study subjects}

Across the study period we enrolled 602 patients. The median time to analgesia was $82 \mathrm{~min}$ (IQR $45-151 \mathrm{~min}$ ); analgesia was provided to $180(30 \%)$ patients within $60 \mathrm{~min}$ of being triaged. Of the 602 patients, 271 (45\%) had cognitive impairment documented and verified (SIS 4 or less). We therefore present the results in two groups: an intention to treat analysis of the 602 patients, and a sensitivity analysis of the 271 patients.

\section{Intention to treat}

There were $323(54 \%)$ patients at intervention sites and 279 (46\%) at non-intervention sites. Table 1 describes patient details, showing considerable baseline differences between the groups despite the randomisation process. Patients at the intervention sites were older, more likely to arrive by ambulance and had a different fracture pattern than those at control sites.

Intervention sites were also almost twice as likely to apply a low triage urgency code to patients (43\% vs $25 \%$ of patients with ATS 4), which was statistically significant $\left(X^{2}, p=0.001\right)$. Intervention sites documented pain scores more frequently 


\begin{tabular}{|c|c|c|c|c|}
\hline Factor & $\begin{array}{l}\text { Intervention } \\
\mathrm{n}=323\end{array}$ & $\begin{array}{l}\text { Control } \\
n=279\end{array}$ & p Value & Adjusted value \\
\hline Female gender & $229(71 \%)$ & $206(74 \%)$ & 0.423 & \\
\hline Age (median, IQR) & $86(79-90)$ & $83(74-89)$ & 0.002 & \\
\hline Fracture site & & & $<0.001$ & \\
\hline Femur & $164(51 \%)$ & $143(51 \%)$ & & \\
\hline Tibia/fibula & $13(4 \%)$ & $14(5 \%)$ & & \\
\hline Radius & $29(9 \%)$ & $35(12 \%)$ & & \\
\hline Humerus & $29(9 \%)$ & $56(20 \%)$ & & \\
\hline Other fracture & $39(12 \%)$ & $19(7 \%)$ & & \\
\hline No fracture & 49 (15\%) & $12(4 \%)$ & & \\
\hline Arrive by ambulance & $285(89 \%)$ & $212(76 \%)$ & 0.001 & \\
\hline Prehospital analgesia & $175(54 \%)$ & $152(55 \%)$ & 0.086 & \\
\hline Triage score & & & 0.001 & \\
\hline ATS 1-2 & $14(5 \%)$ & $19(7 \%)$ & & \\
\hline ATS 3 & $170(53 \%)$ & $191(68 \%)$ & & \\
\hline ATS 4 & $139(43 \%)$ & $69(25 \%)$ & & \\
\hline First pain score median (IQR) $\dagger$ & $5(3-8)$ & $5(3-8)$ & & \\
\hline Time to analgesia median (IQR) & $83(48-158)$ & $82(41-147)$ & 0.42 & 0.74 \\
\hline Analgesia <60 min & $90(28 \%)$ & $90(32 \%)$ & 0.190 & 0.910 \\
\hline No analgesia & $38(12 \%)$ & $25(9 \%)$ & 0.262 & \\
\hline Admitted to hospital & $268(86 \%)$ & $235(86 \%)$ & 0.964 & \\
\hline
\end{tabular}

*Adjusted for age, fracture type, arrival mode to ED and triage category.

TPAINAD for intervention sites, VAS for control sites.

ATS, Australasian triage scale; PAINAD, Pain Assessment in Advanced Dementia; VAS, visual analogue scale.

$(p=0.03)$, but patients were no more likely to receive analgesia within $60 \mathrm{~min}(\mathrm{p}=0.190)$ when compared with control sites.

On univariate analysis, there was no difference in the time to analgesia between the intervention and control groups (median 83 (IQR 48-158) $\mathrm{min}$ vs $82(41-147) \mathrm{min}, \mathrm{p}=0.42)$. After adjusting for age, fracture type, arrival mode and triage category in a Cox regression model, there was no significant difference in time to analgesia between the two groups (HR 0.97, 95\% CI 0.80 to 1.17 , p 0.74 ).

When time to analgesia was dichotomised based on whether analgesia was received within $60 \mathrm{~min}, 28 \%$ of intervention and $32 \%$ of control patient $(\mathrm{p}=0.190)$ received analgesia within this benchmark. In a binary logistic model, again adjusting for baseline imbalances at the sites, no difference in the proportion receiving analgesia within $60 \mathrm{~min}$ was found $(\mathrm{p}=0.91)$. Overall, 63 patients $(10 \%)$ did not receive an analgesic by ambulance officers or in the ED. There was no difference between sites $(p=0.90)$. The majority $(n=503 ; 83.5 \%)$ of patients required surgery and hospital admission.

\section{Sensitivity analysis}

Of the 271 patients with cognitive impairment, 157 (58.0\%) were enrolled from intervention sites and 114 from control sites (42\%) (table 2). Of these, 136 (87.0\%) had a PAINAD completed with a median PAINAD score of 2 (IQR 0-4.0). As seen in the larger cohort, patients in the intervention group were older and more likely to have a low triage acuity.

At the intervention sites, patients received analgesia a median of $13 \mathrm{~min}$ sooner, but it was not statistically significant $(90 \mathrm{vs}$ $103 \mathrm{~min}, \mathrm{p}=0.62$ ). Only $22 \%$ of intervention and $23 \%$ of control patients received analgesia within 60 min of arrival $(p=0.92)$. No difference for time to analgesia between the groups was found after adjusting for covariates (HR 0.87 , 95\% CI 0.63 to $1.20, \mathrm{p}=0.40)$.

\section{DISCUSSION}

In this randomised controlled trial, we did not find a statistically significant difference for time to analgesia with use of PAINAD. While the education programme delivered across sites may have contributed to the sensitivity analysis findings, our results confirm substantial analgesic delay continues and highlights the difficulty of providing timely analgesia to older people with cognitive impairment and an acutely painful condition. This is another wake up call to emergency practitioners concerning analgesic provision in vulnerable populations.

There were considerable baseline differences between the groups despite the randomisation process. Patients at the intervention sites were older, more likely to arrive by ambulance and had a different fracture pattern than those at control sites. Of particular note, intervention sites were also almost twice as likely to apply a low triage urgency code to patients ( $43 \%$ vs $25 \%$ of patients with ATS 4 ). The lower triage codes may have had an impact on the outcomes of this study as code allocation has been shown to impact on time to analgesia. ${ }^{2627}$ In this study, routine triage processes occurred, although research suggests that pain in cognitively impaired people is often under-recognised. Consequently, the findings may have been different had the triage nurse used the PAINAD tool.

EDs are committed to pain management despite studies reporting analgesic delay for people with cognitive impairment. ${ }^{4}$ For this trial, PAINAD was implemented based on the assumption that better recognition and measurement of pain intensity would improve analgesic delivery. While overcrowding, age, triage category and gender have been identified as predictors for analgesic delay, no study has tested the assumption that observational pain assessment tools improve time to analgesia. PAINAD has been tested but always with a focus on pain assessment rather than medication delivery. ${ }^{12} 18$ This study highlights that a tool alone is not sufficient to change practice and that individual judgement and/or the organisational environment may also be a 


\begin{tabular}{|c|c|c|c|c|}
\hline Factor & $\begin{array}{l}\text { Intervention site } \\
\mathrm{n}=157\end{array}$ & $\begin{array}{l}\text { Control site } \\
n=114\end{array}$ & $\mathrm{p}$ Value & Adjust $p$ Value* \\
\hline Female gender & $110(70 \%)$ & $82(72 \%)$ & 0.739 & \\
\hline Age (median, IQR) & $87(84-91)$ & $85(81-90)$ & 0.043 & \\
\hline Fracture site & & & 0.026 & \\
\hline Femur & $95(61 \%)$ & $72(63 \%)$ & & \\
\hline Tibia/fibula & $2(1 \%)$ & $1(1 \%)$ & & \\
\hline Radius & $9(6 \%)$ & $5(4 \%)$ & & \\
\hline Humerus & $10(6 \%)$ & $20(18 \%)$ & & \\
\hline Other fracture & $17(11 \%)$ & $8(7 \%)$ & & \\
\hline No fracture & $24(15 \%)$ & $8(7 \%)$ & & \\
\hline Arrive by ambulance & $150(95 \%)$ & $101(89 \%)$ & 0.03 & \\
\hline Prehospital analgesia & $75(49 \%)$ & $71(64 \%)$ & 0.02 & \\
\hline Triage score & & $<0.001$ & & \\
\hline ATS 4 & $70(45 \%)$ & $19(17 \%)$ & & \\
\hline First pain score (median, IQR) & $5(2-8)$ & $5(2-8)$ & & \\
\hline Time to analgesia (median, IQR) & $90(113)$ & $103(168)$ & 0.62 & 0.40 \\
\hline Analgesia within $60 \mathrm{~min}$ & $35(22 \%)$ & $26(23 \%)$ & 0.92 & \\
\hline No analgesia & $26(10 \%)$ & $37(11 \%)$ & 0.343 & \\
\hline Admitted to hospital & $131(87 \%)$ & $104(91 \%)$ & 0.255 & \\
\hline
\end{tabular}

*Adjusted for age, fracture type, arrival mode to ED and triage category.

factor influencing clinician behaviour. Consequently, the use of screening tools may only be one factor in achieving behaviour change.

The introduction of PAINAD and concurrent nursing education showed a small, although clinically meaningful, improvement in the time to analgesia for a subgroup of older people with verified cognitive impairment. However, the overall analgesic time exceeded a clinically acceptable pain management response. In our study, the findings suggest that the implementation of an intervention, such as PAINAD, requires a coordinated transdisciplinary approach, interprofessional education and contextually relevant resources to support clinician behavioural change. ${ }^{28}$ In comparison to the large array of clinical trials comparing different types of drugs in the management of acute pain, there is a paucity of randomised trial evidence for system changes to improve analgesic provision and no trial evidence for system measures to improve analgesia in people with cognitive impairment. ${ }^{29}$

The most common adult pain assessment practice in ED is to use a verbal rating scale or visual analogue scale, ${ }^{16}$ and yet for cognitively impaired patients this is often inadequate. While Australian pain management literature supports the use of pain assessment tools, there is no policy recommendation for adults unable to self-report. ${ }^{29} 30$ ED pain management for this vulnerable group may improve through a combination of policy development focused on pain assessment and management. ${ }^{31}$

PAINAD has been tested internationally, ${ }^{172}$ but never in such a complex, undifferentiated and high acuity setting as the ED. However, PAINAD is quick and simple to use $(<1 \mathrm{~min})$, is a reliable indicator of pain intensity (0-10 pain score), assesses physiological and psychological pain symptoms ${ }^{13}$ and is usable in the ED. This was supported by a substudy by our team involving emergency nurse focus groups $(n=36)$, which identified that PAINAD was preferred when compared with The Abbey Pain Scale, Doloplus-2 and Pain Assessment Checklist for Seniors with Limited Ability to Communicate. ${ }^{6}$

This study highlights, however, that implementation of an evidence-based pain assessment tool is complex and that EDs may require individual multifactorial implementation considerations such as cultural, transdisciplinary and human factors to improve pain management of people with cognitive impairment. More importantly, the findings highlight two challenges; not just the imperative to improve suboptimal care for an obvious painful condition, but the necessity to detect pain in people with cognitive impairment, especially dementia, presenting with a range of conditions and injuries.

There are a number of limitations with this study. Missing data in small numbers of patients, which may have resulted from competing nurse workload, could have influenced study findings. EDs were invited to enrol in the study so self-nomination may have influenced findings. The small number of clusters may have accounted for the difference in baseline populations and failure to reach statistical significance. The screening of suspected long bone fractures by nurses, using the SIS, may have been influenced by workload and introduced bias into the study. A multidisciplinary team approach may have altered the findings. Hence, a larger powered multidisciplinary study that uses independent research staff to collect data is needed. Cognitive impairment was not independently verifiable in over half the patients and this may have led to sample bias. The study did not determine the appropriateness of the analgesic medication and this is an area for further investigation.

In this multi-centre cluster randomised controlled trial, the use of the PAINAD tool did not result in a statistically significant reduction in time to analgesia in older people with suspected long bone fracture and cognitive impairment. The long delays to analgesia observed in both study arms suggest further trials are warranted and highlighted that pain management is complex when communication barriers exist.

Contributors MF, LC and GA conceived the study, designed the trial and obtained the research funding. MF, LC and GA supervised the conduct of the trial and data collection. MF oversaw recruitment of participating sites and managed the quality of data collection. GA provided statistical advice on the study design, sample and analysis of the data. MF, LC and GA were involved in the drafting and revision of the manuscript. MF takes responsibility for the paper as a whole.

Funding This research was supported by the Emergency Care Institute and the Agency for Clinical Innovation (ACI/D12/1275) New South Wales. Neither body has had any role in the conduct of the research nor the preparation of the manuscript.

Competing interests None declared. 
Ethics approval Approval was obtained from the hospitals' health districts' Human Research Ethics Committees (HREC 1212-430M) and operated according to the guidelines of the National Health and Medical Research Council of Australia.

Provenance and peer review Not commissioned; externally peer reviewed. Data sharing statement According to ethical requirements data sharing is not available.

(c) Article author(s) (or their employer(s) unless otherwise stated in the text of the article) 2018. All rights reserved. No commercial use is permitted unless otherwise expressly granted.

\section{REFERENCES}

1 Samaras N, Chevalley T, Samaras D, et al. Older patients in the emergency department: a review. Ann Emerg Med 2010;56:261-9.

2 Arendts G, Fry M. Factors associated with delay to opiate analgesia in emergency departments. J Pain 2006;7:682-6.

3 Hugenschmidt CE, Sink KM. No pain, functional gain: the importance of pain management in older adults with cognitive impairment. Pain 2015;156:1377-8.

4 Fry M, Arendts G, Chenoweth L, et al. Cognitive impairment is a risk factor for delayed analgesia in older people with long bone fracture: a multicenter exploratory study. Int Psychogeriatr 2015;27:323-8.

5 Rodger KT, Greasley-Adams C, Hodge Z, et al. Expert opinion on the management of pain in hospitalised older patients with cognitive impairment: a mixed methods analysis of a national survey. BMC Geriatr 2015;15:56.

6 Fry M, Arendts G, Chenoweth L. Emergency nurses' evaluation of observational pain assessment tools for older people with cognitive impairment. J Clin Nurs 2017:26:1281-90.

7 Grant PS. Analgesia delivery in the ED. Am J Emerg Med 2006;24:806-9.

8 Timmons S, O'Shea E, O'Neill D, et al. Acute hospital dementia care: results from a national audit. BMC Geriatr 2016;16:113.

9 DeWaters T, Popovich J, Faut-Callahan M. An evaluation of clinical tools to measure pain in older people with cognitive impairment. Br I Community Nurs 2003;8:226-34.

10 Paulson CM, Monroe T, Mion LC. Pain assessment in hospitalized older adults with dementia and delirium. J Gerontol Nurs 2014;40:10-15.

11 Husebo BS, Strand LI, Moe-Nilssen R, et al. Who suffers most? dementia and pain in nursing home patients: a cross-sectional study. J Am Med Dir Assoc 2008;9:427-33.

12 Lukas A, Niederecker T, Günther I, et al. Self- and proxy report for the assessment of pain in patients with and without cognitive impairment: experiences gained in a geriatric hospital. Z Gerontol Geriatr 2013;46:214-21.

13 DeWaters T, Faut-Callahan M, McCann JJ, et al. Comparison of self-reported pain and the PAINAD scale in hospitalized cognitively impaired and intact older adults after hip fracture surgery. Orthop Nurs 2008;27:21-8.

14 National Palliative Care Program. The PMG kit for Aged care. Canberra: Australian Government Department of Health and Ageing, 2007.
15 Warden V, Hurley AC, Volicer L. Development and psychometric evaluation of the Pain Assessment in Advanced Dementia (PAINAD) scale. J Am Med Dir Assoc 2003:4:9-15.

16 Bergman CL. Emergency nurses' perceived barriers to demonstrating caring when managing adult patients' pain. J Emerg Nurs 2012;38:218-25.

17 Jordan A, Hughes J, Pakresi M, et al. The utility of PAINAD in assessing pain in a UK population with severe dementia. Int I Geriatr Psychiatry 2011;26:118-26.

18 Lukas A, Barber JB, Johnson P, et al. Observer-rated pain assessment instruments improve both the detection of pain and the evaluation of pain intensity in people with dementia. Eur J Pain 2013;17:1558-68.

19 Herr K, Coyne PJ, Key T, et al. Pain assessment in the nonverbal patient: position statement with clinical practice recommendations. Pain Manag Nurs 2006;7:44-52.

20 Ellis-Smith C, Evans CJ, Bone AE, et al. Measures to assess commonly experienced symptoms for people with dementia in long-term care settings: a systematic review. BMC Med 2016;14:38.

21 Mosele M, Inelmen EM, Toffanello ED, et al. Psychometric properties of the pain assessment in advanced dementia scale compared to self assessment of pain in elderly patients. Dement Geriatr Cogn Disord 2012;34:38-43.

22 Liu JY, Briggs M, Closs SJ. The psychometric qualities of four observational pain tools (OPTs) for the assessment of pain in elderly people with osteoarthritic pain. J Pain Symptom Manage 2010;40:582-98.

23 Costardi D, Rozzini L, Costanzi C, et al. The Italian version of the pain assessment in advanced dementia (PAINAD) scale. Arch Gerontol Geriatr 2007;44:175-80.

24 Callahan CM, Unverzagt FW, Hui SL, et al. Six-item screener to identify cognitive impairment among potential subjects for clinical research. Med Care 2002;40:771-81.

25 Holdgate A, Asha S, Craig J, et al. Comparison of a verbal numeric rating scale with the visual analogue scale for the measurement of acute pain. Emerg Med 2003;15:441-6.

26 Ducharme J, Tanabe P, Homel P, et al. The influence of triage systems and triage scores on timeliness of ED analgesic administration. Am J Emerg Med 2008;26:867-73.

27 Guéant S, Taleb A, Borel-Kühner J, et al. Quality of pain management in the emergency department: results of a multicentre prospective study. Eur $J$ Anaesthesiol 2011;28:97-105.

28 Galvin R, Gilleit Y, Wallace E, et al. Adverse outcomes in older adults attending emergency departments: a systematic review and meta-analysis of the identification of seniors at risk (ISAR) screening tool. Age Ageing 2017;46:179-186.

29 Schug S, Palmer G, Scott D, et al. eds. Acute pain management: scientific evidence. 4th ed: Australian and New Zealand College of Anaesthetists and Faculty of Pain Medicine, 2015.

30 Australian and New Zealand College of Anaesthetists and Faculty of Pain Medicine. Acute pain management: scientific evidence. Melbourne: Australian and New Zealand College of Anaesthetists, 2005

31 Fry M, Bennetts S, Huckson S. An Australian audit of ED pain management patterns. J Emerg Nurs 2011;37:269-74.

32 Pinto MC, Minson FP, Lopes AC, et al. Cultural adaptation and reproducibility validation of the brazilian portuguese version of the Pain Assessment in Advanced Dementia (PAINAD-Brazil) scale in non-verbal adult patients. Einstein 2015;13:14-19. 